\author{
Ch. Ashyralyev, B. Ozturk
}

Gumushane University, Gumushane, Turkey

(E-mail: charyyar@gumushane.edu.tr)

\title{
Some approximations of second order derivatives complex-valued functions
}

\begin{abstract}
In this paper, we generalize the well known finite difference method to compute derivatives of real valued function to approximate of second order complex derivatives $w_{z z}$ and $w_{\overline{z z}}$ for complex-valued function $w$. Exploring different combinations of terms, we derive several approximations to compute the second order derivatives of complex-valued function. Several second order of accuracy finite differences to calculate derivatives are proposed. Error analysis in test examples is carried out by using Matlab program.
\end{abstract}

Keywords: finite difference, approximation, complex-valued function, approximation formulas.

\section{Introduction}

Boundary value problems for equations with complex-valued functions and partial derivatives with respect to complex variables have important applications in various areas of mathematical modeling of real physical processes [1-6]. The theory of finite difference method in case of real valued function and its applications to solve boundary value problems for partial differential equations is described in [7]. In [8-10], a complex step method for computing derivatives of real valued functions by introducing a complex step in a strict sense is developed. Several finite differences to compute first order derivatives of complex valued function discussed in [7].

Let $C$ be a set of complex numbers, let $\Omega_{1}, \Omega_{2} \subset C$, let $\omega: \Omega_{1} \rightarrow \Omega_{2}$ be a complex-valued function. For each $z=x+i y \in \Omega_{1}$ its image $\omega(z)=\omega(x, y) \in \Omega_{2}$ can be rewritten as $u(x, y)+i v(x, y)$ by introducing pair of real-valued two-dimensional functions $u$ and $v$. Second derivatives $w_{z z}$ and $w_{\overline{z z}}$ at point $z=x+i y$ are defined by

$$
\begin{aligned}
& \omega_{z z}(x, y)=\frac{1}{2}\left(\frac{\partial \omega_{z}}{\partial x}-i \frac{\partial \omega_{z}}{\partial y}\right)=\frac{1}{4}\left(\frac{\partial^{2} \omega}{\partial x^{2}}-i \frac{\partial^{2} \omega}{\partial y \partial x}-i \frac{\partial^{2} \omega}{\partial x \partial y}-\frac{\partial^{2} \omega}{\partial y^{2}}\right) ; \\
& \omega_{\bar{z} \bar{z}}(x, y)=\frac{1}{2}\left(\frac{\partial \omega_{\bar{z}}}{\partial x}+i \frac{\partial \omega_{\bar{z}}}{\partial y}\right)=\frac{1}{4}\left(\frac{\partial^{2} \omega}{\partial x^{2}}+i \frac{\partial^{2} \omega}{\partial y \partial x}+i \frac{\partial^{2} \omega}{\partial x \partial y}-\frac{\partial^{2} \omega}{\partial y^{2}}\right) .
\end{aligned}
$$

\section{Approximation of second order derivatives}

Theorem 1. Assume that the functions $\frac{\partial^{4} u}{\partial y \partial x^{3}}, \frac{\partial^{6} u}{\partial y^{3} \partial x^{3}}, \frac{\partial^{4} v}{\partial y \partial x^{3}}, \frac{\partial^{6} v}{\partial y^{3} \partial x^{3}}$ are continuous and bounded on $\Omega_{1}, h$ and $\tau$ are positive and sufficiently small numbers. Then, the following second order of accuracy approximate formulas for $\omega_{z z}$ are valid:

$$
\begin{aligned}
& \omega_{z z}(x, y)=\frac{1}{4 h^{2}} \omega(x+h, y)+\left(-\frac{1}{2 h^{2}}+\frac{1}{2 \tau^{2}}\right) \omega(x, y)+\frac{1}{4 h^{2}} \omega(x-h, y)- \\
& -\frac{i}{8 h \tau} \omega(x+h, y+\tau)+\frac{i}{8 h \tau} \omega(x-h, y+\tau)+\frac{i}{8 h \tau} \omega(x+h, y-\tau)- \\
& -\frac{i}{8 h \tau} \omega(x-h, y-\tau)-\frac{1}{4 \tau^{2}} \omega(x, y+\tau)-\frac{1}{4 \tau^{2}} \omega(x, y-\tau)+O\left(h^{2}+\tau^{2}\right) \\
& x+i y, x \pm h+i y, x+h+i(y \pm \tau), x-h+i(y \pm \tau) \in \Omega_{1} ;
\end{aligned}
$$




$$
\begin{aligned}
& \omega_{z z}(x, y)=-\frac{1}{4 h^{2}} \omega(x+3 h, y)+\frac{1}{h^{2}} \omega(x+2 h, y)-\frac{5}{4 h^{2}} \omega(x+h, y)+ \\
& +\left(\frac{1}{2 h^{2}}-\frac{1}{2 \tau^{2}}\right) \omega(x, y)-\frac{i}{8 h \tau} \omega(x+h, y+\tau)+\frac{i}{8 h \tau} \omega(x-h, y+\tau)+ \\
& +\frac{i}{8 h \tau} \omega(x+h, y-\tau)-\frac{i}{8 h \tau} \omega(x-h, y-\tau)+\frac{1}{4 \tau^{2}} \omega(x, y+3 \tau)- \\
& -\frac{1}{\tau^{2}} \omega(x, y+2 \tau)+\frac{5}{4 \tau^{2}} \omega(x, y+\tau)+O\left(h^{2}+\tau^{2}\right) \\
& x+3 h+i y, x+2 h+i y, x+h+i y, x+i y \in \Omega_{1} ; \\
& x+h+i(y \pm \tau), x+i(y+3 \tau), x+i(y+2 \tau), x+i(y+\tau) \in \Omega_{1} \\
& \omega_{z z}(x, y)=-\frac{1}{4 h^{2}} \omega(x-3 h, y)+\frac{1}{h^{2}} \omega(x-2 h, y)-\frac{5}{4 h^{2}} \omega(x-h, y)+ \\
& +\left(\frac{1}{2 h^{2}}-\frac{1}{2 \tau^{2}}\right) \omega(x, y)-\frac{i}{8 h \tau} \omega(x+h, y+\tau)+\frac{i}{8 h_{1} \tau} \omega(x-h, y+\tau)+ \\
& +\frac{i}{8 h \tau} \omega(x+h, y-\tau)-\frac{i}{8 h \tau} \omega(x-h, y-\tau)+\frac{1}{4 \tau^{2}} \omega(x, y-3 \tau)- \\
& -\frac{1}{\tau^{2}} \omega(x, y-2 \tau)+\frac{5}{4 \tau^{2}} \omega(x, y-\tau)+O\left(h^{2}+\tau^{2}\right) \\
& x-3 h+i y, x-2 h+i y, x-h+i y, x+i y, x-h+i(y+\tau), \in \Omega_{1} ; \\
& x+h+i(y \pm \tau), x+i(y-3 \tau), x+i(y-2 \tau), x+i(y-\tau) \in \Omega_{1} \\
& \omega_{z z}(x, y)=-\frac{1}{4 h^{2}} \omega(x+3 h, y)+\frac{1}{h^{2}} \omega(x+2 h, y)-\frac{5}{4 h^{2}} \omega(x+h, y)+ \\
& +\left(\frac{1}{2 h^{2}}-\frac{1}{2 \tau^{2}}\right) \omega(x, y)-\frac{i}{8 h \tau} \omega(x+h, y+\tau)+\frac{i}{8 h \tau} \omega(x-h, y+\tau)+ \\
& +\frac{i}{8 h \tau} \omega(x+h, y-\tau)-\frac{i}{8 h \tau} \omega(x-h, y-\tau)+\frac{1}{4 \tau^{2}} \omega(x, y-3 \tau)- \\
& -\frac{1}{\tau^{2}} \omega(x, y-2 \tau)+\frac{5}{4 \tau^{2}} \omega(x, y-\tau)+O\left(h^{2}+\tau^{2}\right) \\
& x+3 h+i y, x+2 h+i y, x+h+i y, x+i y, x+h+i(y \pm \tau) \in \Omega_{1} ; \\
& x-h+i(y \pm \tau), x+i(y-\tau), x+i(y-2 \tau), x+i(y-3 \tau) \in \Omega_{1} ; \\
& \omega_{z z}(x, y)=-\frac{1}{4 h^{2}} \omega(x-3 h, y)+\frac{1}{h^{2}} \omega(x-2 h, y)-\frac{5}{4 h^{2}} \omega(x-h, y)+ \\
& +\left(\frac{1}{2 h^{2}}-\frac{1}{2 \tau^{2}}\right) \omega(x, y)-\frac{i}{8 h \tau} \omega(x+h, y+\tau)+\frac{i}{8 h_{1} \tau} \omega(x-h, y+\tau)+ \\
& +\frac{i}{8 h \tau} \omega(x+h, y-\tau)-\frac{i}{8 h \tau} \omega(x-h, y-\tau)+\frac{1}{4 \tau^{2}} \omega(x, y+3 \tau)- \\
& -\frac{1}{\tau^{2}} \omega(x, y+2 \tau)+\frac{5}{4 \tau^{2}} \omega(x, y+\tau)+O\left(h^{2}+\tau^{2}\right) \\
& x-3 h+i y, x-2 h+i y, x-h+i y, x+i y, x+h+i(y \pm \tau) \in \Omega_{1} ; \\
& x-h+i(y \pm \tau), x+i(y+\tau), x+i(y+2 \tau), x+i(y+3 \tau) \in \Omega_{1} .
\end{aligned}
$$

Proof. By using Taylor decomposition formula for $\frac{\partial^{2} u}{\partial x^{2}}, \frac{\partial^{2} v}{\partial x^{2}}, \frac{\partial^{2} u}{\partial y^{2}}, \frac{\partial^{2} v}{\partial y^{2}}$ at point $(x, y) \in \Omega_{1}$ we have that there exist real numbers $c_{1}, c_{2}, d_{1}, d_{2}$ such that 


$$
\begin{aligned}
& \frac{\partial^{2} u}{\partial x^{2}}(x, y)=\frac{u(x+h, y)-2 u(x, y)+u(x-h, y)}{h^{2}}+\frac{\partial^{3} u}{\partial x^{3}}\left(c_{1}, y\right) \frac{h^{2}}{6} ; \\
& \frac{\partial^{2} v}{\partial x^{2}}(x, y)=\frac{v(x+h, y)-2 v(x, y)+v(x-h, y)}{h^{2}}+\frac{\partial^{3} v}{\partial x^{3}}\left(c_{2}, y\right) \frac{h^{2}}{6} ; \\
& \frac{\partial^{2} u}{\partial y^{2}}(x, y)=\frac{u(x, y+\tau)-2 u(x, y)+u(x, y-\tau)}{\tau^{2}}+\frac{\partial^{3} u}{\partial y^{3}}\left(x, d_{1}\right) \frac{\tau^{2}}{6} ; \\
& \frac{\partial^{2} v}{\partial y^{2}}(x, y)=\frac{v(x, y+\tau)-2 v(x, y)+v(x, y-\tau)}{\tau^{2}}+\frac{\partial^{3} v}{\partial y^{3}}\left(x, d_{2}\right) \frac{\tau^{2}}{6} .
\end{aligned}
$$

Thus

$$
\begin{aligned}
& \frac{\partial^{2} \omega}{\partial x^{2}}(x, y)=\frac{\omega(x+h, y)-2 \omega(x, y)+\omega(x-h, y)}{h^{2}}+\left(\frac{\partial^{3} u}{\partial x^{3}}\left(c_{1}, y\right)+i \frac{\partial^{3} v}{\partial x^{3}}\left(c_{2}, y\right)\right) \frac{h^{2}}{6} \\
& \frac{\partial^{2} \omega}{\partial y^{2}}(x, y)=\frac{\omega(x, y+\tau)-2 \omega(x, y)+\omega(x, y-\tau)}{\tau^{2}}+\left(\frac{\partial^{3} u}{\partial y^{3}}\left(x, d_{1}\right)+i \frac{\partial^{3} v}{\partial y^{3}}\left(x, d_{2}\right)\right) \frac{\tau^{2}}{6} .
\end{aligned}
$$

Applying Taylor decomposition formula for $\frac{\partial u}{\partial x}$ at points $(x, y+\tau)$ and $(x, y-\tau)$, we have that there exists $c_{3}$ such that

$$
\begin{aligned}
& \frac{\partial u}{\partial x}(x, y+\tau)=\frac{u(x+h, y+\tau)-u(x-h, y+\tau)}{2 h}+\frac{\partial^{3} u}{\partial x^{3}}\left(c_{3}, y+\tau\right) \frac{h^{2}}{6} ; \\
& \frac{\partial u}{\partial x}(x, y-\tau)=\frac{u(x+h, y-\tau)-u(x-h, y-\tau)}{2 h}+\frac{\partial^{3} u}{\partial x^{3}}\left(c_{3}, y-\tau\right) \frac{h^{2}}{6} .
\end{aligned}
$$

By Taylor decomposition formula for $\frac{\partial^{2} u}{\partial y \partial x}$ at point $(x, y)$, we have

$$
\frac{\partial^{2} u}{\partial y \partial x}(x, y)=\frac{\frac{\partial u}{\partial x}(x, y+\tau)-\frac{\partial u}{\partial x}(x, y-\tau)}{2 \tau}+\frac{\partial^{4} u}{\partial y^{3} \partial x}\left(x, d_{3}\right) \frac{\tau^{2}}{6}
$$

for some constant $d_{3}$ between $y-\tau$ and $y+\tau$. From (8) and (9) it follows that

$$
\begin{aligned}
& \frac{\partial^{2} u}{\partial y \partial x}(x, y)=\frac{u(x+h, y+\tau)-u(x-h, y+\tau)-u(x+h, y-\tau)+u(x-h, y-\tau)}{4 h \tau}+ \\
& +\frac{1}{2 \tau}\left[\frac{\partial^{3} u}{\partial x^{3}}\left(c_{3}, y+\tau\right) \frac{h^{2}}{6}-\frac{\partial^{3} u}{\partial x^{3}}\left(c_{3}, y-\tau\right) \frac{h^{2}}{6}\right]+\frac{\partial^{4} u}{\partial y^{3} \partial x}\left(x, d_{3}\right) \frac{\tau^{2}}{6} .
\end{aligned}
$$

Since

$$
\frac{1}{2 \tau}\left[\frac{\partial^{3} u}{\partial x^{3}}\left(c_{3}, y+\tau\right)-\frac{\partial^{3} u}{\partial x^{3}}\left(c_{3}, y-\tau\right)\right]=\frac{\partial^{4} u}{\partial y \partial x^{3}}\left(c_{3}, y\right)+\frac{\partial^{6} u}{\partial y^{3} \partial x^{3}}\left(c_{3}, d_{3}\right) \frac{\tau^{2}}{6},
$$

we have

$$
\begin{gathered}
\frac{\partial^{2} u}{\partial y \partial x}(x, y)=\frac{u(x+h, y+\tau)-u(x-h, y+\tau)-u(x+h, y-\tau)+u(x-h, y-\tau)}{4 h \tau}+ \\
+O\left(h^{2}+\tau^{2}\right) .
\end{gathered}
$$

In the similar manner it can be obtained that

$$
\begin{gathered}
\frac{\partial^{2} v}{\partial y \partial x}(x, y)=\frac{v(x+h, y+\tau)-v(x-h, y+\tau)-v(x+h, y-\tau)+v(x-h, y-\tau)}{4 h \tau}+ \\
+O\left(h^{2}+\tau^{2}\right) .
\end{gathered}
$$

Therefore, from (1), (7), (11), and (12) we get (2).

From Taylor decomposition formula it can be showed that there exist numbers $c_{4}, c_{5}, d_{4}, d_{5}$ such that

$$
\begin{aligned}
x-h< & c_{4}, c_{5}<x+3 h, y-\tau<d_{4}, d_{5}<y+3 \tau ; \\
\frac{\partial^{2} \omega}{\partial x^{2}}(x, y)= & \frac{-\omega(x+3 h, y)+4 \omega(x+2 h, y)-5 \omega(x+h, y)+2 \omega(x, y)}{h^{2}}+ \\
& +\left(\frac{\partial^{3} u}{\partial x^{3}}\left(c_{4}, y\right)+i \frac{\partial^{3} v}{\partial x^{3}}\left(c_{5}, y\right)\right) \frac{h^{2}}{6} \\
\frac{\partial^{2} \omega}{\partial y^{2}}(x, y) & =\frac{-\omega(x, y+3 \tau)+4 \omega(x, y+2 \tau)-5 \omega(x, y+\tau)+2 \omega(x, y)}{\tau^{2}}+ \\
& +\left(\frac{\partial^{3} u}{\partial y^{3}}\left(x, d_{4}\right)+i \frac{\partial^{3} v}{\partial y^{3}}\left(x, d_{5}\right)\right) \frac{\tau^{2}}{6} .
\end{aligned}
$$


Formula (3) follows from (1), (13), (11), and (12).

By Taylor decomposition formula, we can get that there exist numbers $c_{6}, c_{7}, d_{6}, d_{7}$ such that

$$
\begin{aligned}
x-3 h< & c_{6}, c_{7}<x+h, y-3 \tau<d_{6}, d_{7}<y+\tau ; \\
\frac{\partial^{2} \omega}{\partial x^{2}}(x, y)= & \frac{-\omega(x-3 h, y)+4 \omega(x-2 h, y)-5 \omega(x-h, y)+2 \omega(x, y)}{h^{2}}+ \\
& +\left(\frac{\partial^{3} u}{\partial x^{3}}\left(c_{6}, y\right)+i \frac{\partial^{3} v}{\partial x^{3}}\left(c_{7}, y\right)\right) \frac{h^{2}}{6} ; \\
\frac{\partial^{2} \omega}{\partial y^{2}}(x, y)= & \frac{-\omega(x, y-3 \tau)+4 \omega(x, y-2 \tau)-5 \omega(x, y-\tau)+2 \omega(x, y)}{\tau^{2}}+ \\
& +\left(\frac{\partial^{3} u}{\partial y^{3}}\left(x, d_{6}\right)+i \frac{\partial^{3} v}{\partial y^{3}}\left(x, d_{7}\right)\right) \frac{\tau^{2}}{6} .
\end{aligned}
$$

So, from (1), (14), (11), and (12) we can obtain (4).

By Taylor decomposition formula, we can prove that there exist numbers $c_{8}, c_{9}, d_{8}, d_{9}$ such that

$$
\begin{gathered}
x-h<c_{8}, c_{9}<x+3 h, y-3 \tau<d_{8}, d_{9}<y+\tau \\
\frac{\partial^{2} \omega}{\partial x^{2}}(x, y)=\frac{-\omega(x+3 h, y)+4 \omega(x+2 h, y)-5 \omega(x+h, y)+2 \omega(x, y)}{h^{2}}+ \\
+\left(\frac{\partial^{3} u}{\partial x^{3}}\left(c_{8}, y\right)+i \frac{\partial^{3} v}{\partial x^{3}}\left(c_{9}, y\right)\right) \frac{h^{2}}{6} ; \\
\frac{\partial^{2} \omega}{\partial y^{2}}(x, y)=\frac{-\omega(x, y-3 \tau)+4 \omega(x, y-2 \tau)-5 \omega(x, y-\tau)+2 \omega(x, y)}{\tau^{2}}+ \\
+\left(\frac{\partial^{3} u}{\partial y^{3}}\left(x, d_{1}\right)+i \frac{\partial^{3} v}{\partial y^{3}}\left(x, d_{2}\right)\right) \frac{\tau^{2}}{6} .
\end{gathered}
$$

Hence, (1), (15), (11), and (12) give us (5).

In the similar manner we show that there exist $c_{10}, c_{11}, d_{10}, d_{11}$ such that

$$
\begin{aligned}
x-3 h & <c_{10}, c_{11}<x, y<d_{10}, d_{11}<y+3 \tau \\
\frac{\partial^{2} \omega}{\partial x^{2}}(x, y)= & \frac{-\omega(x-3 h, y)+4 \omega(x-2 h, y)-5 \omega(x-h, y)+2 \omega(x, y)}{h^{2}}+ \\
& +\left(\frac{\partial^{3} u}{\partial x^{3}}\left(c_{10}, y\right)+i \frac{\partial^{3} v}{\partial x^{3}}\left(c_{11}, y\right)\right) \frac{h^{2}}{6} \\
\frac{\partial^{2} \omega}{\partial y^{2}}(x, y)= & \frac{-\omega(x, y+3 \tau)+4 \omega(x, y+2 \tau)-5 \omega(x, y+\tau)+2 \omega(x, y)}{\tau^{2}}+ \\
& +\left(\frac{\partial^{3} u}{\partial y^{3}}\left(x, d_{10}\right)+i \frac{\partial^{3} v}{\partial y^{3}}\left(x, d_{11}\right)\right) \frac{\tau^{2}}{6}
\end{aligned}
$$

Finally, (1), (16), (11), and (12) give us (6). The proof of Theorem 1 is complete.

In similar manner it can established the following statement.

Theorem 2. Assume that the functions $\frac{\partial^{4} u}{\partial y \partial x^{3}}, \frac{\partial^{6} u}{\partial y^{3} \partial x^{3}}, \frac{\partial^{4} v}{\partial y \partial x^{3}}, \frac{\partial^{6} v}{\partial y^{3} \partial x^{3}}$ are continuous and bounded on $\Omega_{1}, h$ and $\tau$ are positive numbers. Then, the following second order of accuracy approximate formulas for $\omega_{\bar{z} \bar{z}}$ are valid:

$$
\begin{aligned}
& \omega_{\bar{z} \bar{z}}(x, y)=\frac{1}{4 h^{2}} \omega(x+h, y)+\left(-\frac{1}{2 h^{2}}+\frac{1}{2 \tau^{2}}\right) \omega(x, y)+\frac{1}{4 h^{2}} \omega(x-h, y)+ \\
& +\frac{i}{8 h \tau} \omega(x+h, y+\tau)-\frac{i}{8 h \tau} \omega(x-h, y+\tau)-\frac{i}{8 h \tau} \omega(x+h, y-\tau)+ \\
& +\frac{i}{8 h \tau} \omega(x-h, y-\tau)-\frac{1}{4 \tau^{2}} \omega(x, y+\tau)-\frac{1}{4 \tau^{2}} \omega(x, y-\tau)+O\left(h^{2}+\tau^{2}\right) \\
& x+i y, x \pm h+i y, x+h+i(y \pm \tau), x-h+i(y \pm \tau) \in \Omega_{1}
\end{aligned}
$$




$$
\begin{aligned}
& \omega_{\bar{z} \bar{z}}(x, y)=-\frac{1}{4 h^{2}} \omega(x+3 h, y)+\frac{1}{h^{2}} \omega(x+2 h, y)-\frac{5}{4 h^{2}} \omega(x+h, y)+ \\
& +\left(\frac{1}{2 h^{2}}-\frac{1}{2 \tau^{2}}\right) \omega(x, y)+\frac{i}{8 h \tau} \omega(x+h, y+\tau)-\frac{i}{8 h \tau} \omega(x-h, y+\tau)- \\
& -\frac{i}{8 h \tau} \omega(x+h, y-\tau)+\frac{i}{8 h \tau} \omega(x-h, y-\tau)+\frac{1}{4 \tau^{2}} \omega(x, y+3 \tau)- \\
& -\frac{1}{\tau^{2}} \omega(x, y+2 \tau)+\frac{5}{4 \tau^{2}} \omega(x, y+\tau)+O\left(h^{2}+\tau^{2}\right) \\
& x+3 h+i y, x+2 h+i y, x+h+i y, x+i y \in \Omega_{1} ; \\
& x+h+i(y \pm \tau), x+i(y+3 \tau), x+i(y+2 \tau), x+i(y+\tau) \in \Omega_{1} ; \\
& \omega_{\bar{z} \bar{z}}(x, y)=-\frac{1}{4 h^{2}} \omega(x-3 h, y)+\frac{1}{h^{2}} \omega(x-2 h, y)-\frac{5}{4 h^{2}} \omega(x-h, y)+ \\
& +\left(\frac{1}{2 h^{2}}-\frac{1}{2 \tau^{2}}\right) \omega(x, y)+\frac{i}{8 h \tau} \omega(x+h, y+\tau)-\frac{i}{8 h_{1} \tau} \omega(x-h, y+\tau)- \\
& -\frac{i}{8 h \tau} \omega(x+h, y-\tau)+\frac{i}{8 h \tau} \omega(x-h, y-\tau)+\frac{1}{4 \tau^{2}} \omega(x, y-3 \tau)- \\
& -\frac{1}{\tau^{2}} \omega(x, y-2 \tau)+\frac{5}{4 \tau^{2}} \omega(x, y-\tau)+O\left(h^{2}+\tau^{2}\right) \\
& x-3 h+i y, x-2 h+i y, x-h+i y, x+i y, x-h+i(y+\tau), \in \Omega_{1} ; \\
& x+h+i(y \pm \tau), x+i(y-3 \tau), x+i(y-2 \tau), x+i(y-\tau) \in \Omega_{1} \\
& \omega_{\bar{z} \bar{z}}(x, y)=-\frac{1}{4 h^{2}} \omega(x+3 h, y)+\frac{1}{h^{2}} \omega(x+2 h, y)-\frac{5}{4 h^{2}} \omega(x+h, y)+ \\
& +\left(\frac{1}{2 h^{2}}-\frac{1}{2 \tau^{2}}\right) \omega(x, y)+\frac{i}{8 h \tau} \omega(x+h, y+\tau)-\frac{i}{8 h \tau} \omega(x-h, y+\tau)- \\
& -\frac{i}{8 h \tau} \omega(x+h, y-\tau)+\frac{i}{8 h \tau} \omega(x-h, y-\tau)+\frac{1}{4 \tau^{2}} \omega(x, y-3 \tau)- \\
& -\frac{1}{\tau^{2}} \omega(x, y-2 \tau)+\frac{5}{4 \tau^{2}} \omega(x, y-\tau)+O\left(h^{2}+\tau^{2}\right) \\
& x+3 h+i y, x+2 h+i y, x+h+i y, x+i y, x+h+i(y \pm \tau) \in \Omega_{1} ; \\
& x-h+i(y \pm \tau), x+i(y-\tau), x+i(y-2 \tau), x+i(y-3 \tau) \in \Omega_{1} ; \\
& \omega_{\bar{z} \bar{z}}(x, y)=-\frac{1}{4 h^{2}} \omega(x-3 h, y)+\frac{1}{h^{2}} \omega(x-2 h, y)-\frac{5}{4 h^{2}} \omega(x-h, y)+ \\
& +\left(\frac{1}{2 h^{2}}-\frac{1}{2 \tau^{2}}\right) \omega(x, y)+\frac{i}{8 h \tau} \omega(x+h, y+\tau)-\frac{i}{8 h_{1} \tau} \omega(x-h, y+\tau)- \\
& -\frac{i}{8 h \tau} \omega(x+h, y-\tau)+\frac{i}{8 h \tau} \omega(x-h, y-\tau)+\frac{1}{4 \tau^{2}} \omega(x, y+3 \tau)- \\
& -\frac{1}{\tau^{2}} \omega(x, y+2 \tau)+\frac{5}{4 \tau^{2}} \omega\left(x, y+h_{2}\right)+O\left(h^{2}+\tau^{2}\right) ; \\
& x-3 h+i y, x-2 h+i y, x-h+i y, x+i y, x+h+i(y \pm \tau) \in \Omega_{1} ; \\
& x-h+i(y \pm \tau), x+i(y+\tau), x+i(y+2 \tau), x+i(y+3 \tau) \in \Omega_{1} .
\end{aligned}
$$




\section{Numerical results}

In this section, we give numerical results for the second order of accuracy finite differences to calculate the second derivatives with respect to complex variables in test example by using Matlab program. Let $\Omega=\{z \mid z=x+i y,-1 \leq x \leq 1,-1 \leq y \leq 1\}, w(z)=z^{2} \bar{z}+\cos (z)+\sin (\bar{z})$. The set of grid points are defined by

$$
\Omega_{h, \tau}=\left\{z_{k, m}=x_{k}+i y_{m}, x_{k}=(k-1) h, k=\overline{1, N+1} ; \quad y_{m}=(m-1) \tau, m=\overline{1, M+1}\right\},
$$

$h=\frac{2}{N}, \quad \tau=\frac{2}{M}$.

Let $S=\{0,1, \ldots N\}, Q=\{0,1, \ldots M\}$. Denote by

$$
\begin{aligned}
& I^{(1)}=S-\{0\}, J^{(1)}=Q-\{0\} ; \\
& I^{(2)}=S-\{0, N-1, N\}, J^{(2)}=Q-\{0, M-1, M\} ; \\
& I^{(3)}=S-\{0,1,2, N\}, J^{(3)}=Q-\{0,1,2, M\} ; \\
& I^{(4)}=S-\{0, N-1, N\}, J^{(4)}=Q-\{0,1,2\} ; \\
& I^{(5)}=S-\{0,1,2\}, J^{(5)}=Q-\{0,1,2, M-1, M\},
\end{aligned}
$$

a set of indices.

In Table 1 an error of corresponding value of the derivative $\omega_{z z}$ is calculated by

$$
\left\|w_{z z}-w_{z z}^{(n)}\right\|_{C\left(\Omega_{h, \tau}\right)}=\max _{k \in I^{(n)}, m \in J^{(n)}}\left|w_{z z}\left(z_{k, m}\right)-w_{z z}^{(n)}\left(z_{k, m}\right)\right|, n=1,2,3,4,5 .
$$

Here $w_{z z}^{(1)}, w_{z z}^{(2)}, w_{z z}^{(3)}, w_{z z}^{(4)}, w_{z z}^{(5)}$ are approximate value of $\omega_{z z}$ by formulas (2), (3), (4), (5), (6), respectively.

In Table 2 an error of corresponding value of the derivative $w_{\bar{z} \bar{z}}$ is calculated by

$$
\left\|w_{\bar{z} \bar{z}}-w_{\bar{z} \bar{z}}^{(n)}\right\|_{C\left(\Omega_{h, \tau}\right)}=\max _{k \in I^{(n)}, m \in J^{(n)}}\left|w_{\bar{z} \bar{z}}\left(z_{k, m}\right)-w_{\bar{z} \bar{z}}^{(n)}\left(z_{k, m}\right)\right|, n=1,2,3,4,5,
$$

where $w_{\bar{z} \bar{z}}^{(1)}, w_{\bar{z} \bar{z}}^{(2)}, w_{\bar{z} \bar{z}}^{(3)}, w_{\bar{z} \bar{z}}^{(4)}, w_{\bar{z} \bar{z}}^{(5)}$ are approximately value of $\omega_{\bar{z} \bar{z}}$ by formulas (17), (18), (19), (20), (21), respectively.

Table 1

Error analysis for $\omega_{z z}$

\begin{tabular}{|c|c|c|c|c|c|}
\hline $\begin{array}{c}\text { Approximation } \\
\text { formula }\end{array}$ & $\mathrm{N}=10$ & $\mathrm{~N}=20$ & $\mathrm{~N}=40$ & $\mathrm{~N}=80$ & $\mathrm{~N}=160$ \\
$\mathrm{M}=10$ & $\mathrm{M}=20$ & $\mathrm{M}=40$ & $\mathrm{M}=80$ & $\mathrm{M}=160$ \\
\hline$(2)$ & $2.02 \times 10^{-5}$ & $1.39 \times 10^{-6}$ & $9.10 \times 10^{-8}$ & $5.83 \times 10^{-9}$ & $3.70 \times 10^{-10}$ \\
\hline$(3)$ & $5.49 \times 10^{-3}$ & $8.13 \times 10^{-4}$ & $1.10 \times 10^{-4}$ & $1.44 \times 10^{-5}$ & $1.83 \times 10^{-6}$ \\
\hline$(4)$ & $4.81 \times 10^{-3}$ & $7.60 \times 10^{-4}$ & $1.06 \times 10^{-4}$ & $1.41 \times 10^{-5}$ & $1.82 \times 10^{-6}$ \\
\hline$(5)$ & $5.49 \times 10^{-3}$ & $8.13 \times 10^{-4}$ & $1.10 \times 10^{-4}$ & $1.44 \times 10^{-5}$ & $1.83 \times 10^{-6}$ \\
\hline$(6)$ & $4.81 \times 10^{-3}$ & $7.60 \times 10^{-4}$ & $1.07 \times 10^{-4}$ & $1.41 \times 10^{-5}$ & $1.82 \times 10^{-6}$ \\
\hline
\end{tabular}

Table 2

Error analysis for $\omega_{\bar{z} \bar{z}}$

\begin{tabular}{|c|c|c|c|c|c|}
\hline $\begin{array}{c}\text { Approximation } \\
\text { formula }\end{array}$ & $\begin{array}{c}\mathrm{N}=10 \\
\mathrm{M}=10\end{array}$ & $\begin{array}{c}\mathrm{N}=20 \\
\mathrm{M}=20\end{array}$ & $\begin{array}{c}\mathrm{N}=40 \\
\mathrm{M}=40\end{array}$ & $\begin{array}{c}\mathrm{N}=80 \\
\mathrm{M}=80\end{array}$ & $\begin{array}{c}\mathrm{N}=160 \\
\mathrm{M}=160\end{array}$ \\
\hline$(17)$ & $2.02 \times 10^{-5}$ & $1.39 \times 10^{-6}$ & $9.10 \times 10^{-8}$ & $5.83 \times 10^{-9}$ & $3.70 \times 10^{-10}$ \\
\hline$(18)$ & $5.49 \times 10^{-3}$ & $8.13 \times 10^{-4}$ & $1.10 \times 10^{-4}$ & $1.44 \times 10^{-5}$ & $1.83 \times 10^{-6}$ \\
\hline$(19)$ & $4.81 \times 10^{-3}$ & $7.60 \times 10^{-4}$ & $1.06 \times 10^{-4}$ & $1.41 \times 10^{-5}$ & $1.82 \times 10^{-6}$ \\
\hline$(20)$ & $5.49 \times 10^{-3}$ & $8.13 \times 10^{-4}$ & $1.10 \times 10^{-4}$ & $1.44 \times 10^{-5}$ & $1.83 \times 10^{-6}$ \\
\hline$(21)$ & $4.81 \times 10^{-3}$ & $7.60 \times 10^{-4}$ & $1.07 \times 10^{-4}$ & $1.41 \times 10^{-5}$ & $1.82 \times 10^{-6}$ \\
\hline
\end{tabular}




\title{
Conclusion
}

In the present work, we have generalized the finite difference method to compute derivatives of real valued function to approximate the second order complex derivatives $\omega_{z z}$ and $\omega_{\bar{z} \bar{z}}$ for the complex-valued function $\omega$. Exploring different combinations of terms, we derive several approximations to compute the second order derivatives of complex-valued function. Several second order of accuracy finite differences to calculate derivatives are proposed. The error analysis in test examples is carried out by using Matlab program.

\section{References}

1 Muskhelishvili, N. (1953). Singular Integral Equations. Groningen: Noordhoff International Publishing.

2 Vekua, I. (1962). Generalized Analytic Functions. Oxford: Pergamon Press.

3 Monakhov, V. (1983). Boundary-Value Problems with Free Boundaries for Elliptic Systems of Equations. Translations of Mathematical Monographs. (AMS).

4 Gakhov, F. (1990). Boundary Value Problems. Courier Dover Publications.

5 Ashyralyev, C. (1994). Numerical algorithms of the solution for singular integral equations and their applications in hydrodynamics. Ashgabat: Ylym.

6 Ashyralyyev, C., \& Cakir, Z. (2014). AIP Conference Proceedings, 1611, 73-77.

7 Ashyralyyev, C., \& Ozturk, B. (2018). AIP Conference Proceedings, 1997, 020054.

8 Abreu, D.R., \& Morales, J. (2013). Journal of Computational and Applied Mathematics, 241.

9 Abreu, R. (2013). Complex steps finite differences with applications to seismic problems Ph.D. thesis, Universidad de Granada.

10 Samarskii, A. (2001). The theory of difference schemes. New York: Marcell Dekker, Inc.

\author{
Ч. Ашыралыев, Б. Озтурк
}

\section{Комплексмәнді функциялардың екінші ретті туындылары үшін кейбір жуықтаулар}

\begin{abstract}
Мақалада нақты мәнді функциялардың екінші ретті туындыларын есептеуге арналған белгілі ақырлыайырымдар тәсілі жалпы жағдайда дамытылған. Сол арқылы комплекс мәнді $w$ функциясының екінші ретті туындыларын $w_{z z}, w_{\overline{z z}}$ аппроксимациялауға болады. Терминдердің әртүрлі комбинацияларын зерттеу нәтижесінде комплекс мәнді функцияның екінші ретті туындыларын есептеу үшін бірнеші жуықтау формулалары алынды. Туындыларды есептеу үшін дәлдігі екінші ретті бірнеше ақырлы-айырымдар тәсілі ұсынылды. Тест түріндегі мысалдардағы қателіктерге талдау жасау үшін Matlab бағдарламасы пайдаланылды.
\end{abstract}

Kiлm сөздер: ақырлы-айырымдар тәсілі, аппроксимация, комплекс мәнді функция, жуықтау формулалар.

\author{
Ч. Ашыралыев, Б. Озтурк
}

\section{Некоторые приближения производных второго порядка комплекснозначных функций}

\begin{abstract}
В статье обобщен известный метод конечных разностей для вычисления производных вещественной функции на аппроксимацию комплексных производных второго порядка $w_{z z}$ и $w_{\bar{z} z}$ для комплекснозначной функции $w$. Изучая различные комбинации терминов, получено несколько приближений для вычисления производных второго порядка комплекснозначной функции. Предложены несколько конечных разностей второго порядка точности для вычисления производных. Анализ ошибок в тестовых примерах выполнен с использованием программы Matlab.
\end{abstract}

Ключевые слова: конечная разность, аппроксимация, комплекснозначная функция, формулы приближений. 